\title{
On the Relationship between Active and Passive Methods of Mode Localization for Vibration Confinement
}

\author{
Ahmet S. Yigit \\ Department of Mechanical and Industrial Engineering, Kuwait University, P.O. Box 5969, Safat 13060, Kuwait
}

\begin{abstract}
Recently, some researchers have proposed what is termed as "active mode localization" where all or some of the mode shapes are altered by feedback in such a way that the modes are spatially localized. The resulting closedloop behavior is similar, at least qualitatively, to the phenomenon known as "Anderson localization" or normal mode localization which is caused by irregularities (disorders) in periodic structures and which can be considered as a form of passive confinement. This study is an attempt to develop analogies between active and passive means that achieve mode localization. It is demonstrated that in certain cases a passive system can be considered as a special case of active feedback. It is anticipated that the ideas proposed in this study will be useful in developing systematic methods for integrated structure/control designs.
\end{abstract}

\section{INTRODUCTION}

Most structures are subjected to noise and vibration due to the presence of various sources. These sources often excite unwanted structural resonances which can cause damage or the transmission of vibrational energy to distant parts or regions where they cannot be tolerated. Therefore, it may be of interest to contain the vibrational energy near to the points of excitation, and prevent the propagation of large vibrational amplitudes to other parts of a structure. Such systems include aerospace and ship structures, large communication antennas and flexible robotic manipulators carrying very sensitive payloads. In these cases, it is also desirable to suppress the vibrations at a greater rate at those sensitive regions or stations.

Structures exhibiting the so-called "Anderson localization", or normal mode localization phenomenon typically demonstrate a similar behavior. The presence of irregularities in nominally-periodic structures may inhibit the propagation of vibrations within the structure. The mode localization, which occurs due to the variation of structural parameters or due to the addition of passive devices, is here referred to as "passive mode localization". Thus, Anderson localization can be considered a special case of "uncontrolled' passive mode localization. Over the past decade, there has been considerable interest in the Anderson localization phenomenon in engineering structures. ${ }^{1-3}$ Most of these studies have been concerned with how and why this type of mode localization occurs and investigated ways for predicting the occurrence and the degree of localization in different structures with and without damping. Though this localization must be viewed as catastrophic since it leads to large amplitudes at certain locations or regions of a structure, ${ }^{2}$ the possibility of taking advantage of this phenomenon as a means of passive vibration- isolation, has been suggested by several researchers. Hodges ${ }^{1}$ has pointed out some potential use of vibration confinement similar to damping. Allaei ${ }^{4}$ has investigated the feasibility of developing an efficient vibration-control methodology based on mode localization.
Vakakis ${ }^{5}$ has shown that the impulsive response can be localized in weakly-coupled, geometrically-nonlinear beams. Nayfeh and Hawwa ${ }^{6}$ have suggested use of mode localization as a means to control structural buckling. Since Anderson localization has been shown to occur in nominally periodic structures, most of the above studies were concerned with only periodic structures.

Recently, some researchers have proposed what is termed here as "active mode localization" for confinement of vibrations. Active mode localization is achieved by feedback as opposed to parameter variations. A method, based on applying a distributed feedback for a class of one and two-dimensional structures such as uniform strings and beams, is given in References. ${ }^{7,8}$ The method consists of altering the mode shapes to exponentially-decaying functions of space by feedback. The excitations are kept localized at the place of their origin. The method has been shown to be effective in confining vibrations in a prescribed region by the introduction of a suitable time delay. ${ }^{9}$ A similar method suitable for discrete systems or for systems described by lumped parameter models, such as the Finite Element Method, has been proposed by Choura. ${ }^{10}$

The idea of an active, passive or a hybrid design to yield mode localization has also been proposed by Allaei. ${ }^{11}$ Passive methods involve a suitable change in the design of a structure so that the mode shapes are localized in certain geometric regions of the structural domain, whereas active methods require the use of actuators to induce the localized modes by feedback. In several publications the efficacy of a control strategy based on mode localization has been demonstrated. ${ }^{12-15}$ In a recent publication, Allaei ${ }^{16}$ provided some performance comparisons between the so-called "Vibration Control by Confinement, (VCC)" and more conventional controllers, and concluded that the VCC strategy is a powerful and flexible technique which may offer innovative solutions to some vibration problems.

Another group of researchers have approached the problem as an eigenstructure assignment problem. Shaw and Jayasuriya ${ }^{17}$ have studied the conditions for arbitrary assignment of eigenvectors by state feedback. Song and Jayasu- 